\title{
On a new Wigner-Ville distribution associated with linear canonical transform
}

Hong-Cai Xin ${ }^{1,2}$ and Bing-Zhao Li $i^{1,2^{*}}$

*Correspondence:
li_bingzhao@bit.edu.cn
${ }^{1}$ School of Mathematics and
Statistics, Beijing Institute of
Technology, Beijing, China
${ }^{2}$ Beijing Key Laboratory on MCAACl,
Beijing Institute of Technology,
Beijing, China

Beijing, China

\begin{abstract}
Linear canonical transform as a general integration transform has been considered into Wigner-Ville distribution (WVD) to show more powerful ability for non-stationary signal processing. In this paper, a new WVD associated with linear canonical transform (WVDL) and integration form of WVDL (IWVDL) are presented. First, the definition of $W V D L$ is derived based on new autocorrelation function and some properties are investigated in details. It removes the coupling between time and time delay and lays the foundation for signal analysis and processing. Then, based on the characteristics of WVDL over time-frequency plane, a new parameter estimation method, IWVDL, is proposed for linear modulation frequency (LFM) signal. Two phase parameters of LFM signal are estimated simultaneously and the cross term can be suppressed well by integration operator. Finally, compared with classical WVD, the simulation experiments are carried out to verify its better estimation and suppression of cross term ability. Error analysis and computational cost are discussed to show superior performance compared with other WVD in linear canonical transform domain. The further application in radar imaging field will be studied in the future work.
\end{abstract}

Keywords: Linear canonical transform, Wigner-Ville distribution, Time-frequency analysis, Parameter estimation

\section{Introduction}

The classical Wigner-Ville distribution (WVD), as an important and fundamental tool of time-frequency analysis, has been developed over the years in many engineering systems [1-6]. It can be also viewed as traditional Fourier transform (FT) kernel on autocorrelation function. Linear canonical transform (LCT) is a generalized integral transform of FT and fractional FT (FRFT) and defined as [7-9]

$$
X_{A}(u)=\mathcal{L}_{A}\{x(t)\}(u)=\int_{-\infty}^{+\infty} x(t) K_{A}(u, t) d t
$$

where

$$
K_{A}(u, t)=\left\{\begin{array}{cc}
\frac{1}{\sqrt{i 2 \pi b}} \cdot e^{j\left(\frac{a}{2 b} t^{2}-\frac{1}{b} u t+\frac{d}{2 b} u^{2}\right)}, & b \neq 0 \\
\sqrt{d} \cdot e^{j \frac{c d}{2} u^{2}} \delta(t-d u), & b=0
\end{array}\right.
$$

(c) The Author(s). 2021 Open Access This article is licensed under a Creative Commons Attribution 4.0 International License, which permits use, sharing, adaptation, distribution and reproduction in any medium or format, as long as you give appropriate credit to the original author(s) and the source, provide a link to the Creative Commons licence, and indicate if changes were made. The images or other third party material in this article are included in the article's Creative Commons licence, unless indicated otherwise in a credit line to the material. If material is not included in the article's Creative Commons licence and your intended use is not permitted by statutory regulation or exceeds the permitted use, you will need to obtain permission directly from the copyright holder. To view a copy of this licence, visit http://creativecommons.org/licenses/by/4.0/. 
and parameter matrix $A=\left[\begin{array}{llll}a & b ; & c & d\end{array}\right], a, b, c, d \in \mathbb{R}$ and $|A|=1$. From (1) and (2), LCT can be reduced to be FT, FRFT, or other affine transform when $A$ is chosen specially. LCT plays a major role in non-stationary signal processing, especially for detection and estimation of LFM signals, which has been developed into diverse application areas [10-13]. However, although LCT and WVD are important and effective tools in LFM signal processing, LCT cannot gather signal energy strongly like WVD and only classical WVD does not exploit phase feature of LFM signal fully. As a result, they display the poor performance under low signal-to-noise ratio (SNR) for detection and estimation. In order to improve the performance of LFM signal detection and estimation, therefore, a series of WVD associated with LCT have been proposed by researchers, which are effective way and validated by some works [14-22].

The WVD associated with the LCT (LCWD) was first investigated from generalized transform domain perspective in [14] and the relations among some time-frequency distributions and linear canonical operators have been discussed. Along with this idea, the WVD associated with LCT has been studied in depth and widely [15-20, 22]. The WVD based on LCT (WDL) was first defined in time domain by replacing the kernel of classical FT with kernel of LCT [15]. Based on this notion, some basic properties were also derived. This WDL raised more analysis views of LFM signal in time-linear canonical frequency (LCF) plane and then was applied to estimating parameter of LFM signals. In [16], a new version of LCWD and its moment were investigated, which was applied to first-order optical system. In the same year, a cross-WVD for time-frequency analysis and generalized WVD for the estimation of quadratic frequency modulation signal were presented in LCT domain [17]. Afterwards, unified WVD in the LCT domain (UWDL) was proposed by substituting the classical autocorrelation function with a generalized autocorrelation function in [18], which unifies LCWD and WDL by nine free parameters. In order reduce parameter complexity of UWDL, two special cases of this UWDL were presented by less parameters $[19,20]$. These unified WDL have more robust detection performance of LFM signal in noise environment than those in $[14,15]$ as well as the classical WVD [1]. However, autocorrelation function of LFM signal contains coupling between time and time delay, which is the essential phase characteristics and is not considered in above methods. For this, the author proposed a novel WVD associated with LCT in [21] and some basic properties were derived. This method can remove coupling and make energy distribution a straight line parallel to time axis, which was used to detect LFM signal theoretically. Nevertheless, there are other useful properties and better performance for parameter estimation of LFM signal based on this method that have not been developed.

For this purpose, the definition of WVD associated with LCT (WVDL) is derived by new autocorrelation function in LCT domain. The WVDL is able to represent and acquire characteristics of LFM signal using affine transform advantage of LCT to time-frequency plane. Then, other useful properties [23, 24] are investigated and proved in details including nonlinearity, linear canonical time shift, linear canonical modulation, dilation, reconstruction formula, and convolution. Based on above characteristics of energy distribution, a novel parameter estimation method, integration form of WVDL (IWVDL), is proposed, which estimate two phase coefficients of LFM simultaneously and suppress cross terms effectively for multi-component LFM signal. Finally, to demonstrate superior estimation performance of LFM signal, the simulation experiments are carried out and the estimation error, computation cost and application are discussed. 
The remainder of this paper is organized as follows: Preliminaries summarizes common notations and basic operators and then reviews existing WVD definitions in the Section 2. Section 3 presents a WVDL from a new point of view and derive some properties. Based on this definition, a new parameter estimation method IWVDL is proposed. Section 4 is the results and discussion about estimation performance and application of proposed method. Section 5 concludes this paper.

\section{Preliminaries}

\subsection{Notations and operators}

We will summarize some notations and review some basic operators in this subsection (Table 1).

Time Shift: For a complex signal $x(t) \in L^{2}(\mathbb{R})$, a time shift operator $\mathbf{T}_{s}$ is given as

$$
\mathbf{T}_{s} x(t)=x(t-s)
$$

Modulation: For a complex signal $x(t) \in L^{2}(\mathbb{R})$, a modulation operator $\mathbf{M}_{u_{0}}$ is given as

$$
\mathbf{M}_{u_{0}} x(t)=x(t) \cdot e^{j u_{0} t}
$$

Dilation: For a complex signal $x(t) \in L^{2}(\mathbb{R})$, a dilation operator $\mathbf{D}_{t_{0}}$ is given as

$$
\mathbf{D}_{t_{0}} x(t)=\frac{1}{t_{0}} x\left(t_{0} t\right)
$$

Linear canonical time shift: For a complex signal $x(t) \in L^{2}(\mathbb{R})$, a linear canonical time shift operator $\mathbf{T}_{s}^{A}$ is given as

$$
\mathbf{T}_{s}^{A} x(t)=x(t-s) e^{-j \frac{a}{b}\left(t-\frac{s}{2}\right) s}
$$

Linear canonical modulation: For a complex signal $x(t) \in L^{2}(\mathbb{R})$, a linear canonical modulation operator $\mathbf{M}_{u_{0}}^{A}$ is given as

$$
\mathbf{M}_{u_{0}}^{A} x(t)=x(t) e^{-j \frac{u_{0}}{b} t}
$$

Convolution $[25,26]$ : For complex signals $x_{1}(t), x_{2}(t) \in L^{2}(\mathbb{R})$, a convolution operator $\circledast$ is given as

$$
x_{1}(t) \circledast x_{2}(t)=\int_{\mathbb{R}} x_{1}\left(t^{\prime}\right) x_{2}\left(t-t^{\prime}\right) d t^{\prime}
$$

\subsection{Wigner-Ville distribution}

The WVD plays a important role in time-frequency representation since it can provide good energy distribution and high resolution for non-stationary signal processing.

Table 1 Some common notation

\begin{tabular}{ll}
\hline Notation & Description \\
\hline $\mathbb{R}$ & The set of real number \\
$*$ & The complex conjugate operator \\
$\circledast$ & The convolution operator of Fourier transform \\
$|a|$ & The modules of $a$ \\
$\delta(t)$ & The continuous-time Dirac function \\
$x(t)$ & The continuous-time signal with finite energy \\
$L^{2}(\mathbb{R})$ & The set of square-integrable function on $\mathbb{R}$ \\
$\mathcal{L}_{A}$ & The LCT operator with parameter matrix $A$ \\
$\langle f(t), g(t)\rangle$ & $L^{2}$-inner product between function $f(t)$ and $g(t)$ \\
\hline &
\end{tabular}


Definition 1. [1, 3] For complex signal $x(t) \in L^{2}(\mathbb{R})$, the classical WVD of $x(t)$ is defined as

$$
W V D(t, \omega)=\int_{\mathbb{R}} x\left(t+\frac{\tau}{2}\right) x^{*}\left(t-\frac{\tau}{2}\right) \cdot e^{-j \tau \omega} d \tau
$$

which is FT of instantaneous autocorrelation function

$$
R(t, \tau)=x\left(t+\frac{\tau}{2}\right) x^{*}\left(t-\frac{\tau}{2}\right)
$$

Motivated by classical WVD and requirement of non-stationary signal processing, a series of novel WVD associated with LCT are proposed to process LFM signal and can be summarized in Table 2. As a matter of fact, above WVDs in LCT are obtained by using new transform kernel, new autocorrelation function, or other methods. As described in the Section 3, they need to improve performance in robust to noise and computational burden for signal processing. Besides, all of them do not fully utilize the characteristics of autocorrelation function of LFM signal and not remove the coupling relationship between time and time delay. Therefore, a newly defined WVD associated with LCT is first proposed in [21], which can remove the coupling and accumulate energy of LFM signal well in the time-LCF plane.

\section{Method}

This section will derive the WVDL from a new perspective and then study some useful properties in details. Based on these, a integration form of WVDL will be proposed to estimate parameters of LFM signal.

\subsection{Definition of WVDL}

It is well known that classical WVD in (9) is Fourier transform of autocorrelation function in (10). In order to obtain new WVDL, we define a new instantaneous autocorrelation function in LCT domain

$$
\begin{aligned}
R^{A}(t, \tau) & =\left[x\left(t+\frac{\tau}{2}\right) e^{j \frac{a}{2 b} t \tau}\right]\left[x^{*}\left(t-\frac{\tau}{2}\right) e^{-j \frac{a}{2 b} t \tau}\right] \\
& =x\left(t+\frac{\tau}{2}\right) x^{*}\left(t-\frac{\tau}{2}\right) e^{j \frac{a}{b} t \tau}
\end{aligned}
$$

Table 2 Existing WVD associated with LCT

\begin{tabular}{l} 
Formula \\
\hline$W_{A}(t, u)=\int_{\mathbb{R}} X_{A}\left(u+\frac{\tau}{2}\right) X_{A}^{*}\left(u-\frac{\tau}{2}\right) e^{-j v \tau} d \tau$ \\
$W_{A}^{X}(t, u)=\int_{\mathbb{R}} X\left(t+\frac{\tau}{2}\right) x^{*}\left(t-\frac{\tau}{2}\right) K_{A}(u, \tau) d \tau$ \\
$W_{A}(t, u)=\int_{\mathbb{R}} X_{A}\left(\frac{u+u^{\prime}}{2}\right) X_{A}^{*}\left(\frac{u+u^{\prime}}{2}\right) e^{j 2 \pi u^{\prime} t} d u^{\prime}$ \\
$W_{x}^{A_{1}, A_{2}, A_{3}}(t, u)=\frac{1}{\sqrt{j 2 \pi b_{3}}} \int_{\mathbb{R}} X_{A_{1}}\left(t+\frac{\tau}{2}\right) X_{A_{2}}^{*}\left(t-\frac{\tau}{2}\right) K_{A_{3}}(u, \tau) d \tau$ \\
where $A_{1}, A_{2}, A_{3}$ are different parameter matrices of $L C T$. \\
$W_{x}^{A_{1}, A_{0}}(t, u)=\frac{1}{\sqrt{j 2 \pi b_{0}}} \int_{\mathbb{R}} X_{A}\left(t+\frac{\tau}{2}\right) x^{*}\left(t-\frac{\tau}{2}\right) K_{A_{0}}(u, \tau) d \tau$ \\
where $A_{0}=\left[a_{0} b_{0}, c_{0}, d_{0}\right]$ is the parameter matrix of $L C T$. \\
$L W D_{A}^{x}(t, u)=2 \int_{\mathbb{R}} X_{A}(\omega+b u) X_{\bar{A}}^{*}(\omega-b u) e^{-j b d u^{2}} K_{A}^{*}(\omega, 2 t) d \omega$ \\
where $\bar{A}=[a-b ;-c d]$. \\
$W D O L_{X}(t, u)=\int_{\mathbb{R}} x\left(t+\frac{\tau}{2}\right) x^{*}\left(t-\frac{\tau}{2}\right) h_{A}(u, t) d \tau$ \\
where $h_{A}=K_{A}(u, \tau) e^{\frac{j}{2 b}\left[a t^{2}+2 t\left(u_{0}-u\right) 2 u\left(d u_{0}-b w_{0}\right)+d u^{2}\right]}$.
\end{tabular}


Applying scaling FT to (11) along with scale time delay $\frac{\tau}{b}$, it can be obtained

$$
\begin{aligned}
& \frac{1}{2 \pi|b|} \int_{\mathbb{R}} R^{A}(t, \tau) \cdot e^{-j \frac{\tau}{b} u} d \tau \\
= & \frac{1}{2 \pi|b|} \int_{\mathbb{R}} x\left(t+\frac{\tau}{2}\right) x^{*}\left(t-\frac{\tau}{2}\right) e^{j \frac{a}{b} t \tau} e^{-j \frac{\tau}{b} u} d \tau
\end{aligned}
$$

According to (11) and (12), a new WVDL can be defined.

Definition 2. [21] For complex signal $x(t) \in L^{2}(\mathbb{R})$, the Wigner-Ville distribution associated with LCT (WVDL) of $x(t)$ is defined as

$$
W V D L_{x}^{A}(t, u)=\frac{1}{2 \pi|b|} \int_{\mathbb{R}} x\left(t+\frac{\tau}{2}\right) x^{*}\left(t-\frac{\tau}{2}\right) \cdot e^{-j \tau\left(\frac{u}{b}-\frac{a t}{b}\right)} d \tau
$$

The $W V D L_{x}^{A}(t, u)$ displays the distribution of signal energy and can be finite support obviously over time-LCF plane, which can provide theoretical foundation for signal detection and estimation. Without loss of generality, assume that $b>0$, so $|b|=b$ and they are not differentiated in the next sections. Particularly, when $A=\left[\begin{array}{ll}0 & ;-1\end{array}\right]$, (13) will reduce to be classical WVD in (9).

It is worth pointing out that the existing other results of WVD in LCT domain [14-16, 18-20] can be regarded as the rotation or affine transform of the classical time-frequency plane. Similarly, there is a relationship between (13) and (9) [21]

$$
W V D L_{x}^{A}(t, u)=\frac{1}{2 \pi|b|} W V D[t,(u-a t) / b]
$$

In the practical engineering, it is finite for observation time to observe and process a system or a signal. Hence, for a LFM signal $x(t)=e^{j\left(f_{0} t+\frac{k}{2} t^{2}\right)}$ with observation time $T_{a}$, its $W V D L_{x}^{A}(t, u)$ can be expressed as

$$
\begin{aligned}
W V D L_{x}^{A}(t, u) & =\frac{1}{2 \pi|b|} \int_{\mathbb{R}} x\left(t+\frac{\tau}{2}\right) x^{*}\left(t-\frac{\tau}{2}\right) \cdot e^{-j \tau\left(\frac{u}{b}-\frac{a t}{b}\right)} d \tau \\
& =\frac{1}{2 \pi|b|} \int_{-T_{a} / 2}^{T_{a} / 2} x\left(t+\frac{\tau}{2}\right) x^{*}\left(t-\frac{\tau}{2}\right) \cdot e^{-j \tau\left(\frac{u}{b}-\frac{a t}{b}\right)} d \tau \\
& =\frac{1}{2 \pi|b|} \int_{-T_{a} / 2}^{T_{a} / 2} e^{j\left(f_{0}+k t\right) \tau} e^{-j \tau\left(\frac{u}{b}-\frac{a t}{b}\right)} d \tau \\
& =\frac{T_{a}}{2 \pi|b|} \operatorname{sinc}\left\{\frac{T_{a}}{2}\left[\left(\frac{u}{b}-f_{0}\right)+\left(\left(\frac{a}{b}+k\right) t\right)\right]\right\}
\end{aligned}
$$

where phase coefficients $f_{0}$ and $k$ are center frequency and modulation frequency rate respectively. From (15), a oblique line is shown in time-LCF plane, which represents the linear relationship between time and LCF. It is worth noting that a straight line paralleling to the time axis is obtained by (15) when $\frac{a}{b}=-k$, which is optimal and pivotal to energy gather and parameter estimation of LFM signal.

\subsection{Properties of WVDL}

Some interesting and basic properties of WVDL have been studied shown in Table 3 [21]. For the sake of completeness of the study on WVDL, there are other useful properties to be derived, which are helpful for analysis of cross term, the relationship of time width and bandwidth, filter design, and other feature analysis of signal. The properties and corresponding formulas are also summarized in Table 4. 
Table 3 Basic properties of new WVDL

\begin{tabular}{ll}
\hline Property & Formula \\
\hline Conjugation symmetry & $W V D L_{x_{1}}^{A}(t, u)=\left[W V D L_{x_{1}}^{A}(t, u)\right]^{*}$, \\
$W V D L_{x_{1}, x_{2}}^{A}(t, u)=\left[W V D L_{x_{1}, x_{2}}^{A}(t, u)\right]^{*}$ & $W V D L_{\mathbf{T}_{s} x}^{A}(t, u)=W V D L_{x}^{A}(t-s, u-a s)$ \\
Time shift & $W V D L_{\mathbf{m}_{u_{0} x}}^{A}(t, u)=\frac{1}{2 \pi} W V D L_{x}^{A}\left(t, u-b u_{0}\right)$ \\
Modulation & $\int_{\mathbb{R}} W V D L_{x}^{A}(t, u) d u=|x(t)|^{2}$ \\
Marginal & $\int_{\mathbb{R}} \int_{\mathbb{R}} W V D L_{x}^{A}(t, u) d u d t=\int_{\mathbb{R}}|x(t)|^{2} d t$ \\
Power & $\int_{\mathbb{R}} \int_{\mathbb{R}} W V D L_{x_{1}}^{A}(t, u)\left[W V D L_{x_{2}}^{A}(t, u)\right]^{*} d u d t$ \\
Moyal's formula & $=\frac{1}{2 \pi|b|}\left|\left\langle x_{1}(t), x_{2}(t)\right\rangle\right|^{2}$ \\
\hline
\end{tabular}

(1) Nonlinearity: Let $W V D L_{x_{1}}^{A}(t, u), W V D L_{x_{2}}^{A}(t, u)$ be WVDLs of complex signal $x_{1}(t), x_{2}(t) \in L^{2}(\mathbb{R})$ and $x_{1}(t), x_{2}(t) \neq 0$ respectively. If $x(t)=x_{1}(t)+x_{2}(t)$, then $W V D L_{x}^{A}$ of $x(t)$ is

$$
W V D L_{x}^{A}(t, u) \neq W V D L_{x_{1}}^{A}(t, u)+W V D L_{x_{2}}^{A}(t, u)
$$

Proof According to the proposed definition of WVDL in (13), we have

$$
\begin{aligned}
& W V D L_{x}^{A}(t, u) \\
= & \frac{1}{2 \pi|b|} \int_{\mathbb{R}}\left[x_{1}\left(t+\frac{\tau}{2}\right)+x_{2}\left(t+\frac{\tau}{2}\right)\right]\left[x_{1}\left(t-\frac{\tau}{2}\right)+x_{2}\left(t-\frac{\tau}{2}\right)\right]^{*} \\
& \cdot e^{-j \tau\left(\frac{u}{b}-\frac{a t}{b}\right)} d \tau \\
= & \frac{1}{2 \pi|b|} \int_{\mathbb{R}}\left[x_{1}\left(t+\frac{\tau}{2}\right) x_{1}^{*}\left(t-\frac{\tau}{2}\right)+x_{2}\left(t+\frac{\tau}{2}\right) x_{2}^{*}\left(t-\frac{\tau}{2}\right)\right. \\
& \left.\quad+x_{1}\left(t+\frac{\tau}{2}\right) x_{2}^{*}\left(t-\frac{\tau}{2}\right)+x_{2}\left(t+\frac{\tau}{2}\right) x_{1}^{*}\left(t-\frac{\tau}{2}\right)\right] \cdot e^{-j \tau\left(\frac{u}{b}-\frac{a t}{b}\right)} d \tau \\
= & W V D L_{x_{1}}^{A}(t, u)+W V D L_{x_{2}}^{A}(t, u)+W V D L_{x_{1} x_{2}}^{A}(t, u)+W V D L_{x_{2} x_{1}}^{A}(t, u)
\end{aligned}
$$

From (17), the output includes two WVDLs of cross terms, which implies nonlinearity in

\begin{tabular}{|c|c|}
\hline Property & Formula \\
\hline Nonlinearity & $W V D L_{x}^{A}(t, u) \neq W V D L_{x_{1}}^{A}(t, u)+W V D L_{x_{2}}^{A}(t, u)$ \\
\hline Linear canonical time shift & $W V D L_{\mathbf{T}_{s}^{A} x}^{A}(t, u)=W V D L_{x}^{A}(t-s, u)$ \\
\hline Linear canonical modulation & $W V D L_{\mathbf{M}_{u_{0}}^{A} x}^{A}(t, u)=W V D L_{x}^{A}\left(t, u-u_{0}\right)$ \\
\hline Dilation & $W V D L_{\mathbf{D}_{t_{0}} x}^{A}(t, u)=\frac{1}{t_{0}} W V D L_{x}^{A_{1}}\left(\frac{t}{t_{0}}, t_{0} u\right)$ \\
\hline Reconstruction formula & 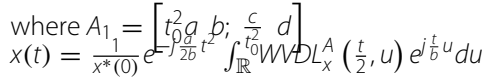 \\
\hline Convolution & $\begin{array}{l}W V D L_{x_{1} \circledast x_{2}}^{A}(t, u)=2 \pi|b| \int_{\mathbb{R}} W V D L_{x_{1} x_{2}}^{A}(w, u) \\
\cdot W V D L_{x_{2} x_{1}}^{A}(t-w, u-a w) d w\end{array}$ \\
\hline
\end{tabular}
(16) holds.

(2) Linear canonical time shift: Let $\operatorname{WVDL}_{x}^{A}(t, u)$ be WVDL of complex signal $x(t) \in$ $L^{2}(\mathbb{R})$, if $\mathbf{T}_{s}^{A}[x(t)]=x(t-s) \cdot e^{-j \frac{a}{b} s\left(t-\frac{s}{2}\right)}$, then

$$
W V D L_{\mathbf{T}_{s}^{A} x}^{A}(t, u)=W V D L_{x}^{A}(t-s, u)
$$

Table 4 Other useful properties of new WVDL 
Proof Based on time shift operator in (6) and definition of WVDL in (13), we have

$$
\begin{aligned}
& W V D L_{\mathbf{T}_{s}^{A} x}^{A}(t, u) \\
= & \frac{1}{2 \pi|b|} \int_{\mathbb{R}}\left[x\left(t-s+\frac{\tau}{2}\right) e^{-j \frac{a}{b} s\left(t-\frac{s}{2}+\frac{\tau}{2}\right)}\right]\left[x\left(t-s-\frac{\tau}{2}\right) e^{-j \frac{a}{b} s\left(t-\frac{s}{2}-\frac{\tau}{2}\right)}\right]^{*} \\
& \cdot e^{-j \tau\left(\frac{u}{b}-\frac{a t}{b}\right)} d \tau \\
= & \frac{1}{2 \pi|b|} \int_{\mathbb{R}} x\left(t-s+\frac{\tau}{2}\right) x^{*}\left(t-s-\frac{\tau}{2}\right) \cdot e^{-j \tau\left(\frac{u-a s}{b}-\frac{a}{b}(t-s)\right)} d \tau \\
= & W V D L_{x}^{A}(t-s, u)
\end{aligned}
$$

This completes the proof.

(3) Linear canonical modulation: Let $W V D L_{x}^{A}(t, u)$ be WVDL of complex signal $x(t) \in$ $L^{2}(\mathbb{R})$, if $\mathbf{M}_{u_{0}}^{A}[x(t)]=x(t) \cdot e^{j \frac{u_{0}}{b} t}$, then

$$
W V D L_{\mathbf{M}_{u_{0}}^{A} x}^{A}(t, u)=W V D L_{x}^{A}\left(t, u-u_{0}\right)
$$

Proof Based on modulation operator in (7) and definition of WVDL in (13), we have

$$
\begin{aligned}
& W V D L_{\mathbf{M}_{u_{0}}^{A} x}^{A}(t, u) \\
= & \frac{1}{2 \pi|b|} \int_{\mathbb{R}}\left[x\left(t+\frac{\tau}{2}\right) e^{j \frac{u_{0}}{b}\left(t+\frac{\tau}{2}\right)}\right]\left[x\left(t-\frac{\tau}{2}\right) e^{j \frac{u_{0}}{b}\left(t-\frac{\tau}{2}\right)}\right]^{*} \cdot e^{-j \tau\left(\frac{u}{b}-\frac{a t}{b}\right)} d \tau \\
= & \frac{1}{2 \pi|b|} \int_{\mathbb{R}} x\left(t+\frac{\tau}{2}\right) x^{*}\left(t-\frac{\tau}{2}\right) \cdot e^{-j \tau\left(\frac{u-b u_{0}}{b}-\frac{a}{b} t\right)} d \tau \\
= & \operatorname{WVDL}_{x}^{A}\left(t, u-b u_{0}\right)
\end{aligned}
$$

Thus, linear canonical modulation property is proved.

(4)Dilation: Let $W V D L_{x}^{A}(t, u)$ be WVDL of complex signal $x(t) \in L^{2}(\mathbb{R})$, if $\mathbf{D}_{t_{0}}[x(t)]=$ $\frac{1}{t_{0}} x\left(t_{0} t\right)$, then

$$
W V D L_{\mathbf{D}_{t_{0}} x}^{A}(t, u)=\frac{1}{t_{0}} W V D L_{x}^{A_{1}}\left(\frac{t}{t_{0}}, t_{0} u\right)
$$

where $A_{1}=\left[\begin{array}{ccc}t_{0}^{2} a & b ; \frac{c}{t_{0}^{2}} & d\end{array}\right]$.

Proof From dilation operator and definition of WVDL in (13), we obtain that

$$
W V D L_{D_{t_{0}} x}^{A}(t, u)=\frac{1}{2 \pi|b|} \frac{1}{t_{0}^{2}} \int_{\mathbb{R}} x\left(\frac{t}{t_{0}}+\frac{\tau}{2 t_{0}}\right) x^{*}\left(\frac{t}{t_{0}}-\frac{\tau}{2 t_{0}}\right) \cdot e^{-j \tau\left(\frac{u}{b}-\frac{a t}{b}\right)} d \tau
$$

Let $\frac{\tau}{t_{0}}=\tau^{\prime},(23)$ can be written as

$$
\begin{aligned}
& W V D L_{D_{t_{0}} x}^{A}(t, u) \\
= & \frac{1}{2 \pi|b|} \frac{1}{t_{0}^{2}} \int_{\mathbb{R}} x\left(\frac{t}{t_{0}}+\frac{\tau^{\prime}}{2}\right) x^{*}\left(\frac{t}{t_{0}}-\frac{\tau^{\prime}}{2}\right) \cdot e^{-j \tau^{\prime}\left(\frac{t_{0} u}{b}-\frac{t_{0}^{2} a}{b} \frac{t}{t_{0}}\right)} d\left(t_{0} \tau^{\prime}\right) \\
= & \frac{1}{2 \pi|b|} \frac{1}{t_{0}} \int_{\mathbb{R}} x\left(\frac{t}{t_{0}}+\frac{\tau^{\prime}}{2}\right) x^{*}\left(\frac{t}{t_{0}}-\frac{\tau^{\prime}}{2}\right) \cdot e^{-j \tau^{\prime}\left(\frac{t_{0} u}{b}-\frac{t_{0}^{2} a}{b} \frac{t}{t_{0}}\right)} d \tau^{\prime} \\
= & \frac{1}{t_{0}} W V D L_{x}^{A_{1}}\left(\frac{t}{t_{0}}, t_{0} u\right)
\end{aligned}
$$

where $A_{1}=\left[t_{0}^{2} a b ; \frac{c}{t_{0}^{2}} d\right]$. This finishes the proof. 
(5) Reconstruction formula: Let $W V D L_{x}^{A}(t, u)$ be WVDL of complex signal $x(t) \in L^{2}(\mathbb{R})$. If initial value $x(0)$ of signal is known and $x(0) \neq 0, b>0$ then

$$
x(t)=\frac{1}{x^{*}(0)} e^{-j \frac{a}{2 b} t^{2}} \int_{\mathbb{R}} W V D L_{x}^{A}\left(\frac{t}{2}, u\right) e^{j \frac{t}{b} u} d u
$$

Proof The newly defined WVDL in (13) can be rewritten as

$$
\begin{aligned}
W V D L_{x}^{A}(t, u) & =\frac{1}{2 \pi|b|} \int_{\mathbb{R}} x\left(t+\frac{\tau}{2}\right) x^{*}\left(t-\frac{\tau}{2}\right) \cdot e^{j \frac{a}{b} \tau t} e^{-j \frac{\tau}{b} u} d \tau \\
& =\frac{1}{2 \pi|b|} \mathcal{F}\{h\}\left(\frac{u}{b}\right)
\end{aligned}
$$

where $\mathcal{F}$ is classical Fourier transform operator, and

$$
h=x\left(t+\frac{\tau}{2}\right) x^{*}\left(t-\frac{\tau}{2}\right) \cdot e^{j \frac{a}{b} \tau t}=\int_{\mathbb{R}} W V D L_{x}^{A}(t, u) e^{j \frac{\tau}{b} u} d u
$$

Let $t=\frac{\tau}{2}$, then $\tau=2 t$. (27) can be reduced

$$
x(2 t) x^{*}(0) e^{j \frac{2 a}{b} t^{2}}=\int_{\mathbb{R}} W V D L_{x}^{A}(t, u) e^{j \frac{2 t}{b} u} d u
$$

Further, let $v=2 t$, then

$$
x(v)=\frac{1}{x^{*}(0)} e^{-j \frac{a}{2 b} v^{2}} \int_{\mathbb{R}} W V D L_{x}^{A}\left(\frac{v}{2}, u\right) e^{j \frac{v}{b} u} d u
$$

Let $t=v$, we have

$$
x(t)=\frac{1}{x^{*}(0)} e^{-j \frac{a}{2 b} t^{2}} \int_{\mathbb{R}} W V D L_{x}^{A}\left(\frac{t}{2}, u\right) e^{j \frac{t}{b} u} d u
$$

Thus, it completes the signal reconstruction.

(6) Convolution: For complex signal $x_{1}, x_{2} \in L^{2}(\mathbb{R})$, then WVDL of $x_{1} \circledast x_{2}$ is

$$
W V D L_{x_{1} \circledast x_{2}}^{A}(t, u)=2 \pi|b| \int_{\mathbb{R}} W V D L_{x_{1} x_{2}}^{A}(w, u) W V D L_{x_{2} x_{1}}^{A}(t-w, u-a w) d w
$$

Proof First, we know

$$
\left[x_{1} \circledast x_{2}\right](t)=\int_{\mathbb{R}} x_{1}\left(t^{\prime}\right) x_{2}\left(t-t^{\prime}\right) d t^{\prime}
$$

According to definition of WVDL, then

$$
\begin{aligned}
& W V D L_{x_{1} \circledast x_{2}}^{A}(t, u) \\
= & \frac{1}{2 \pi|b|} \int_{\mathbb{R}}\left[x_{1} \circledast x_{2}\right]\left(t+\frac{\tau}{2}\right)\left[x_{1} \circledast x_{2}\right]^{*}\left(t-\frac{\tau}{2}\right) \cdot e^{-j \tau\left(\frac{u}{b}-\frac{a t}{b}\right)} d \tau \\
= & \frac{1}{2 \pi|b|} \int_{\mathbb{R}} \int_{\mathbb{R}} x_{1}(r) x_{2}\left(t+\frac{\tau}{2}-r\right) d r \int_{\mathbb{R}} x_{2}^{*}(z) x_{1}^{*}\left(t-\frac{\tau}{2}-z\right) d z \cdot e^{-j \tau\left(\frac{u}{b}-\frac{a t}{b}\right)} d \tau
\end{aligned}
$$


Let $r=w+\frac{p}{2}, z=w-\frac{p}{2}$ and $\tau=p+q$, then (33) can be reduced as

$$
\begin{aligned}
& W V D L_{x_{1} \circledast x_{2}}^{A}(t, u) \\
= & \frac{1}{2 \pi|b|} \int_{\mathbb{R}} \int_{\mathbb{R}} \int_{\mathbb{R}} x_{1}\left(w+\frac{p}{2}\right) x_{2}\left(t-w+\frac{q}{2}\right) x_{2}^{*}\left(w-\frac{p}{2}\right) x_{1}^{*}\left(t-w-\frac{q}{2}\right) \\
= & \frac{1}{2 \pi|b|} \int_{\mathbb{R}} \int_{\mathbb{R}} x_{1}\left(w+\frac{p}{2}\right) x_{2}^{*}\left(w-\frac{q}{2}\right) \cdot e^{-j p\left(\frac{u}{b}-\frac{a t}{b}\right)} d p \\
& \quad \int_{\mathbb{R}} x_{2}\left(t-w+\frac{q}{2}\right) x_{1}^{*}\left(t-w-\frac{q}{2}\right) \cdot e^{-j q\left(\frac{u-a w}{b}-\frac{a}{b}(t-w)\right)} d q d w \\
= & 2 \pi|b| \int_{\mathbb{R}} W V D L_{x_{1} x_{2}}^{A}(w, u) W V D L_{x_{2} x_{1}}^{A}(t-w, u-a w) d w
\end{aligned}
$$

which completes the proof.

\subsection{IWVDL method for LFM signal}

LFM signal plays vital part in many signal processing scenarios like radar, communication, optical, and other fields. In this subsection, a integration form of WVDL (IWVDL) will be proposed for parameter estimation of LFM signal.

For mono-component LFM signal,

$$
x(t)=C e^{j\left(f t+\frac{k}{2} t^{2}\right)}, \quad t \in\left[-\frac{T_{a}}{2}, \frac{T_{a}}{2}\right]
$$

its representation result can be obtained from (15)

$$
W V D L_{x}^{A}(t, u)=\frac{T_{a} C}{2 \pi|b|} \operatorname{sinc}\left\{\frac{T_{a}}{2}\left[\left(\frac{u}{b}-f\right)+\left(\left(k+\frac{a}{b}\right) t\right)\right]\right\}
$$

The WVDL can gather energy of signal in the time-LCF plane, the maximum value of energy distribution is determined by $k=-\frac{a}{b}$. In order to enhance peak, we propose a integral way to WVDL along with time axis

$$
I W V D L_{x}^{A}(u)=\int_{T_{a}} W V D L_{x}^{A}(t, u) d t
$$

Two phase parameters of LFM signal can be acquired simultaneously by the position of energy peak

$$
\left\{\begin{array}{l}
\left\{\frac{a}{b}, u_{0}\right\}=\arg \max _{\frac{a}{b}, u}\left\{I W V D L_{x}^{A}(u)\right\} \\
\hat{k}=-\frac{a}{b} \\
\hat{f}=\frac{u_{0}}{b}
\end{array}\right.
$$

In the observation time $T_{a}$, the multi-component LFM signal is

$$
x(t)=\sum_{i=1}^{L} C_{i} e^{j\left(f_{i} t+\frac{k_{i}}{2}\right) t^{2}} \quad t \in\left[-\frac{T_{a}}{2}, \frac{T_{a}}{2}\right]
$$

where $L$ is the number of component, $C_{i}, f_{i}, k_{i}$ are the amplitude, center frequency and modulation frequency rate of the $i$-th component, respectively. Applying WVDL to (39)

$$
\begin{aligned}
W V D L_{x}^{A}(t, u) & =\frac{1}{2 \pi|b|} \int_{\mathbb{R}} x\left(t+\frac{\tau}{2}\right) x^{*}\left(t-\frac{\tau}{2}\right) \cdot e^{-j \tau\left(\frac{u}{b}-\frac{a t}{b}\right)} d \tau \\
& =\frac{1}{2 \pi|b|} \int_{T_{a}} x\left(t+\frac{\tau}{2}\right) x^{*}\left(t-\frac{\tau}{2}\right) \cdot e^{-j \tau\left(\frac{u}{b}-\frac{a t}{b}\right)} d \tau \\
& =W V D L_{\text {auto }}^{A}(t, u)+W V D L_{\text {cross }}^{A}(t, u)
\end{aligned}
$$


where

$$
W V D L_{\text {auto }}^{A}(t, u)=\frac{T_{a}}{2 \pi|b|} \sum_{i=1}^{L} C_{i}^{2} \operatorname{sinc}\left\{\frac{T_{a}}{2}\left[\left(\frac{u}{b}-f_{i}\right)+\left(\left(k_{i}+\frac{a}{b}\right) t\right)\right]\right\}
$$

and as for the expression of cross terms, please see [21].

To enhance peak and suppress cross term, the integration along time axis is considered into WVDL

$$
I W V D L_{x}^{A}(u)=I W V D L_{\text {auto }}^{A}(u)+I W V D L_{\text {cross }}^{A}(u)
$$

In the parameter search, cross term cannot be integrated actually since they contain $t^{2}$ term in the exponential term and proposed method cannot match them.

\section{Results and discussion}

This section will dedicate to demonstrate the estimation performance of proposed method. The experiments for mono-component and multi-component LFM signal will be first performed. Then, error analysis, computation cost, and application in radar imaging field will be discussed.

\subsection{Results}

\subsubsection{Experiment 1: Mono-component LFM signal}

In the observation time $T_{a}=16 s$, mono-component LFM signal in (35) with $C=1$, $f=1$, and $k=-1$ is shown in Fig. 1a, in which real part and imaginary part are blue solid line and red dotted line respectively. To indicate the characteristics of instantaneous frequency of signal in (35), an oblique line is reproduced by means of phase derivation in the time-frequency plane.

In order to display the feature of matched WVDL for LFM signal, Fig. 2 gives four WVDL results under four parameter matrices. Figure 2a can be viewed as classical WVD since $A_{1}=\left[\begin{array}{lll}0 & -1 ; 1\end{array}\right]$ is chosen, which gives the energy distribution in the classical time-frequency domain. Figure $2 \mathrm{~b}-\mathrm{d}$ are obtained respectively by WVDLs with $A_{2}=[0.5-1 ; 10], A_{1}=[1-1 ; 10], A_{1}=[1.5-1 ; 10]$. From Fig. 2c, proposed WVDL can obtain a straight line paralleling to the time axis, which is the optimal matched LCT domain. Figure 3 is the results by proposed IWVDL method, in which only Fig. 3c obtains

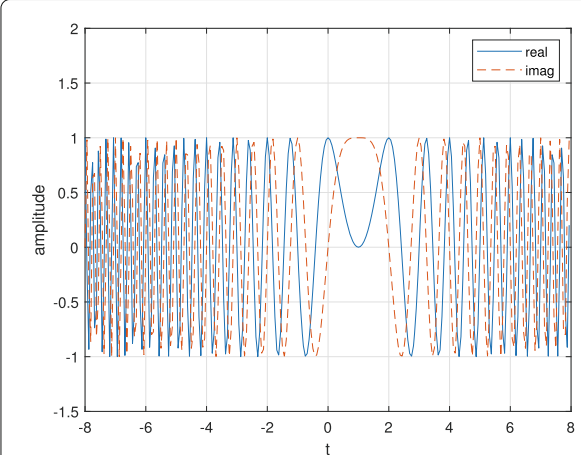

(a) in time domain

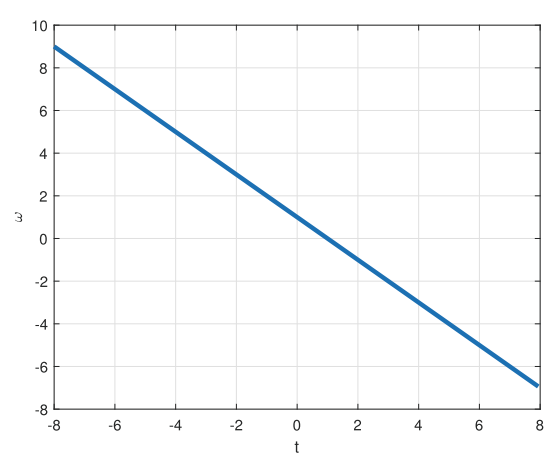

(b) instantaneous frequency

Fig. 1 Mono-component LFM signal 


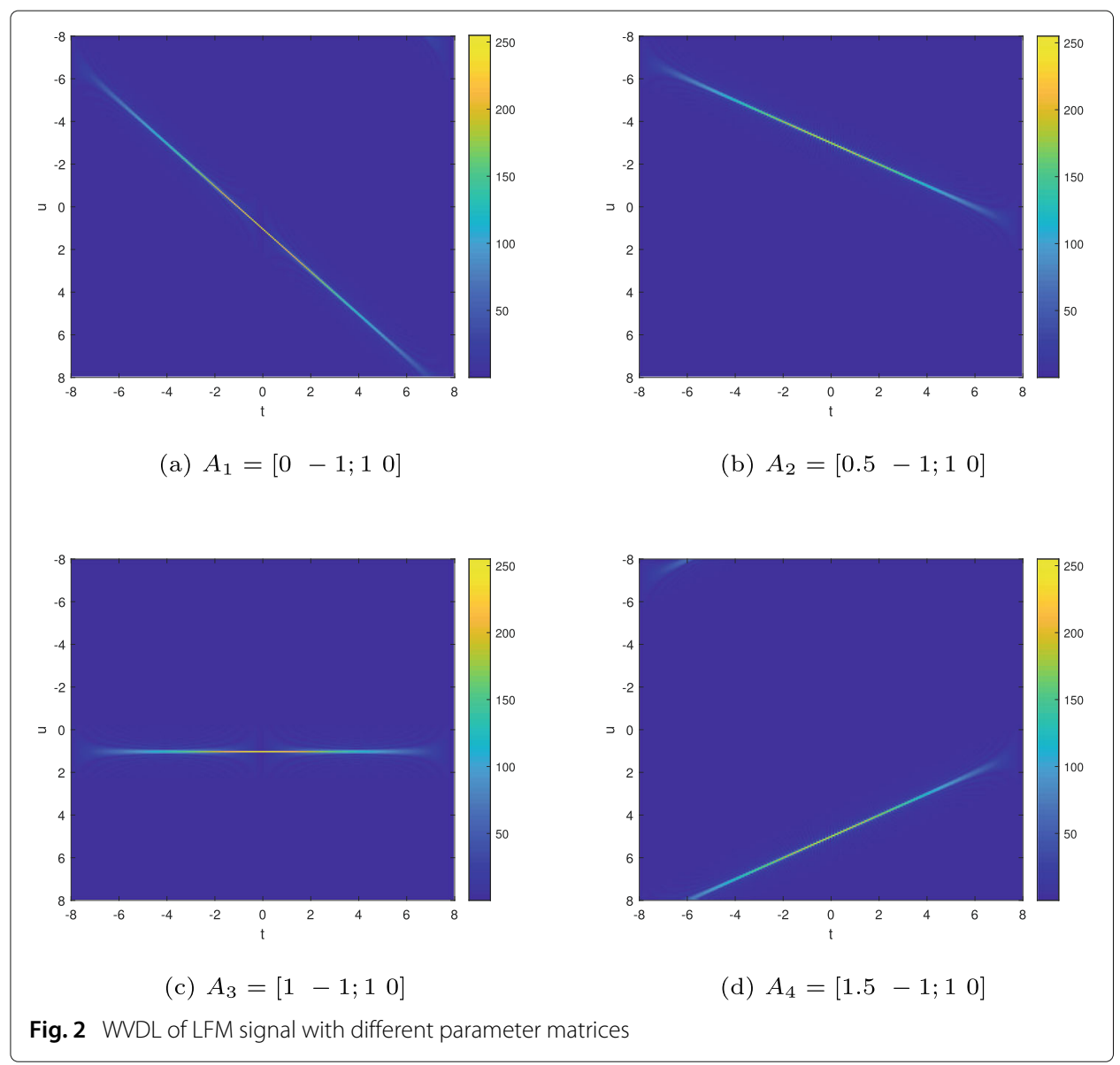

a peak of LFM signal. Similar to other WVD in LCT domain [14-16, 18, 19], the results in the Fig. 2a, b, and d cannot acquire parameters of LFM signal directly in time-LCF plane since they still depend on time axis. Thus, Fig. 3a, b, and $d$ after the integration cannot obtain energy peak of LFM signal. Figures $2 \mathrm{c}$ and $3 \mathrm{c}$ are able to obtain two phase coefficients of LFM signal simultaneously by proposed method.

\subsubsection{Experiment 2: Multi-component LFM signal}

Without loss of generality, two-component LFM signal in (39) when $L=2$ is employed to validate proposed method. In the observation time $T_{a}=16$, the parameters of twocomponent LFM signal are amplitude $C_{1}=C_{2}=1$, phase coefficients $f_{1}=1, f_{2}=-1$, and $k_{1}=-1, k_{2}=0.4$. Figure 4 a shows signal $x(t)$ in time domain by real part and imaginary part.

Figure 5 is classical WVD over time-frequency plane, in which two intersecting lines only imply time-frequency distribution of two components and hardly obtain more special parametric information of signal. Figure 6 are the results by WVDL, in which the matched WVDL of $x_{1}$ is shown and cross term can also not be ignored. Using characteristics of WVDL of LFM signal in time-LCF plane, IWVDL in Fig. 7 works well for suppressing cross term compared with Fig. 7. The parameters of two-component LFM signal can be estimated by peak search in the optimal and matched domain. 


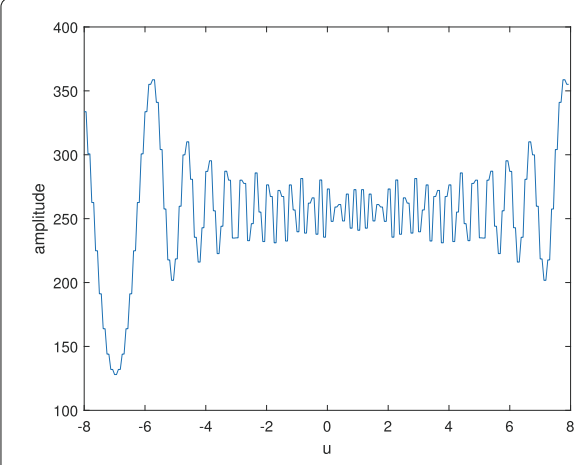

(a) $A_{1}=\left[\begin{array}{lll}0 & -1 ; 1 & 0\end{array}\right]$

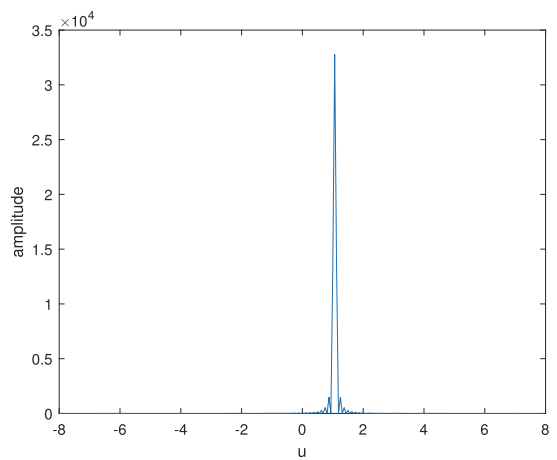

(c) $A_{3}=\left[\begin{array}{ccc}1 & -1 ; 1 & 0\end{array}\right]$

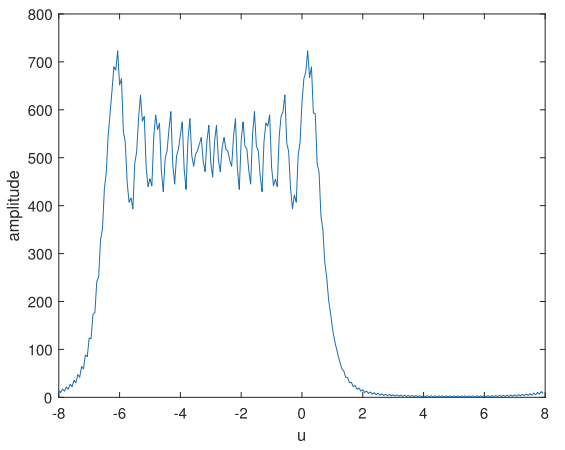

(b) $A_{2}=[0.5-1 ; 10]$

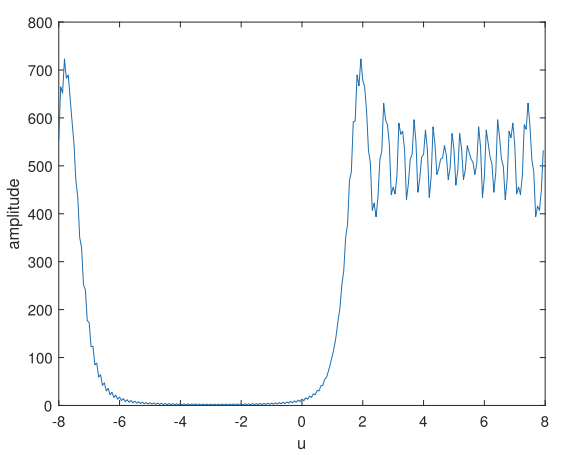

(d) $A_{4}=\left[\begin{array}{lll}1.5 & -1 ; 1 & 0\end{array}\right]$

Fig. 3 IMVDL of LFM signal with different parameter matrices

\subsection{Discussion}

\subsubsection{Error analysis}

In order to validate estimation performance of proposed method, we employ mean square error (MSE) to measured estimation error in different noise environment. The MSE of phase coefficients $f$ and $k$ can be given here [27]

$$
M S E_{f}=10 \log _{10} \frac{1}{N_{\text {trail }}} \sum_{n=1}^{N_{\text {trail }}}\left[\hat{f}_{n}-f\right]^{2}
$$

and

$$
M S E_{k}=10 \log _{10} \frac{1}{N_{\text {trail }}} \sum_{n=1}^{N_{\text {trail }}}\left[\hat{k_{n}}-k\right]^{2}
$$

where $N_{t}$ rail is the number of simulation, $\hat{f}_{n}$ and $\hat{k_{n}}$ are the estimation values of phase coefficients $f$ and $k$ at the $n$th trail.

For existing WVDs in LCT domain [14-16, 18-20], there are some common problems for parameter estimation: (1) do not utilize the phase characteristics of autocorrelation function of LFM signal, (2) do not suppress cross term for multi-component signal, and (3) need other method to further estimate parameters like least square method. Therefore, let us choose a common kind of WVD in LCT domain, the WDL in [15], to be compared to verify the better estimation performance of proposed method. 


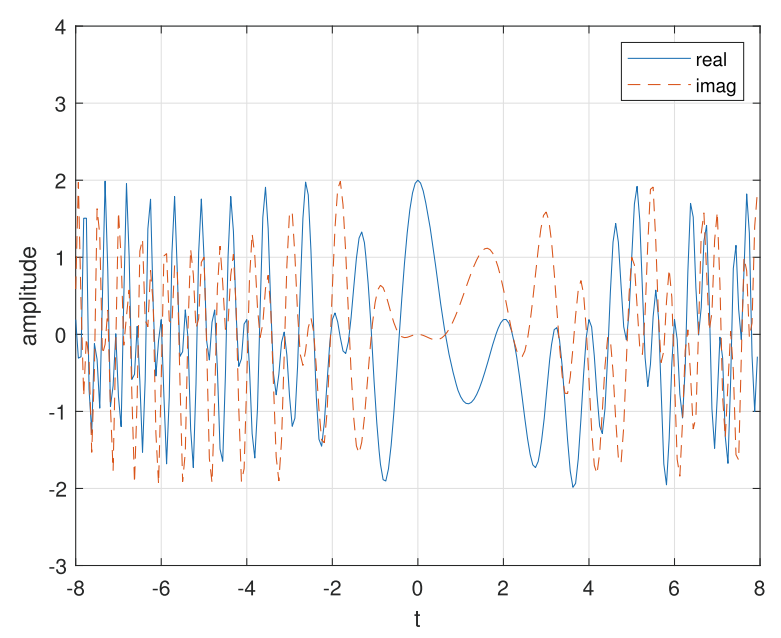

Fig. 4 Two-component LFM signal

For LFM signal with additive white Gaussian noise, 100 Monte Carlo trails will be carried out under low signal-to-noise rate (SNR) $[-15,-5] \mathrm{dB}$. The MSE of different LFM signals are shown in Fig. 8. Figure $8 \mathrm{a}$ is the result for mono-component LFM signal, from which the method based on WDL displays larger error and our method will present higher accuracy for phase coefficients $f$ and $k$ in the $[-15,-5] \mathrm{dB}$. Besides, the estimation errors of multi-component LFM signal are also measured and shown in Fig. 8b. In Fig. 8b, the estimation results based on WDL represent better accuracy in the lower SNR environment but the accuracy does not be improve when SNR increase. Proposed IWVDL method can obtain more accurate parameters when $\mathrm{SNR} \geq-12 \mathrm{~dB}$ and trend to be stable when $\mathrm{SNR} \geq-9 \mathrm{~dB}$. Therefore, our proposed method is more robust against noise and has better estimation performance.

\subsubsection{Computational cost}

For a LFM signal with digital length $N$, the computation cost of classical WVD is $O\left(N^{2} \log _{2} N\right)$ based on fast FT. The computation cost of WDL is $O\left(N^{3}\right)$ introduced by LCT kernel. The proposed IWVDL method contains free parameters of LCT, its computation includes autocorrelation function $N$, WVDL on autocorrelation function $N^{2}$. Assuming

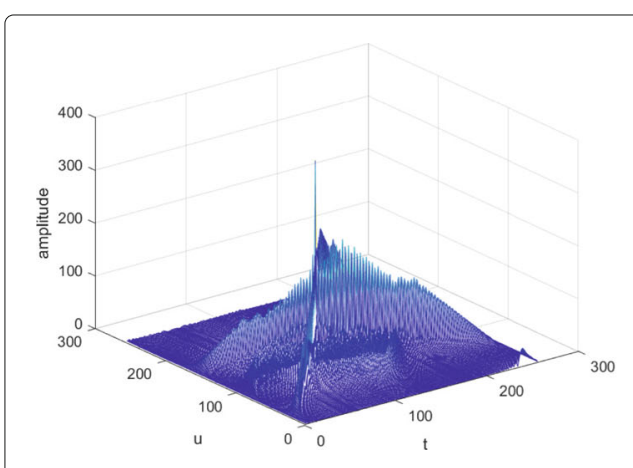

(a) classical WVD

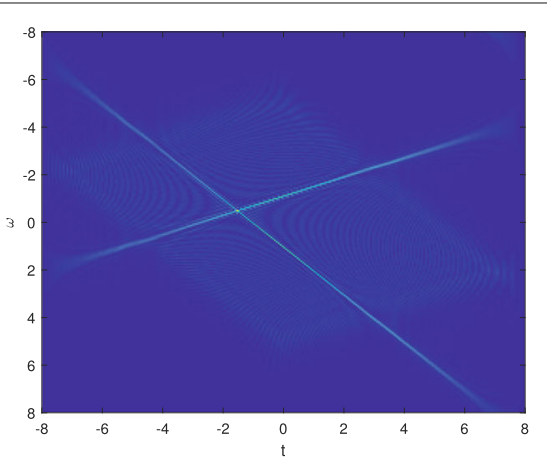

(b) classical WVD in $t-u$ plane

Fig. 5 Classical WVD of two-component LFM signal 


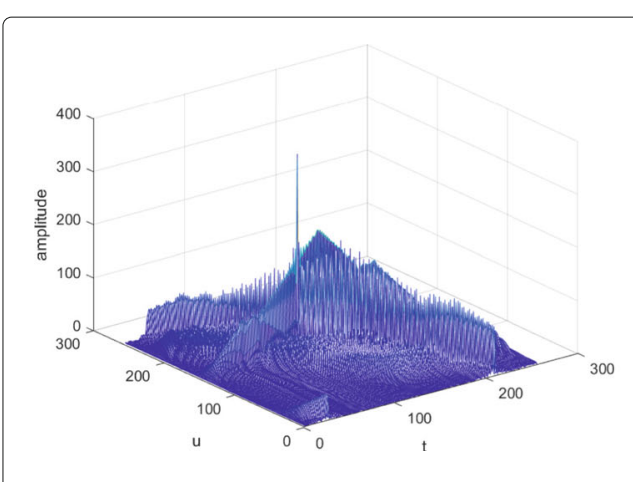

(a) WVDL

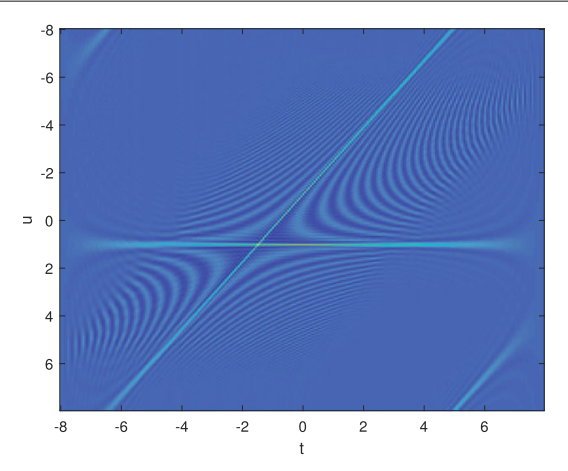

(b) WVDL in $t-u$ plane

Fig. 6 WVDL of two-component LFM signal

search times are $M$; therefore, the computational cost is $O\left(M N^{3}\right)$. There are search procedures for other WVD in LCT domain; their computation become also more complex. For example, unified WVDs associated with LFM have nine or six free parameters, in which burden computation cost have to be faced.

\subsubsection{Application}

The inverse synthetic aperture radar (ISAR) imaging plays an important role for target recognition and classical in the military and civilian areas. The motion of observation target becomes more complex due to complex practical environment, especially the ship target over the sea. Its Doppler is no longer constant and shows time-varying by complex motion [28], which make range-Doppler imaging algorithm based on FT difficult to obtain well-focus ISAR image.

After range compression and motion compensation, the Doppler is generally modeled as LFM signal like (15) according to complex motion of rolling, roll, and yaw over the sea. Therefore, the azimuth echo in a range bin can be modeled as multi-component LFM signal $[29,30]$

$$
x(t)=\sum_{i=1}^{L} C_{i} e^{j\left(a_{i 1} t+\frac{a_{i 2}}{2}\right) t^{2}} \quad t \in\left[-\frac{T_{a}}{2}, \frac{T_{a}}{2}\right]
$$

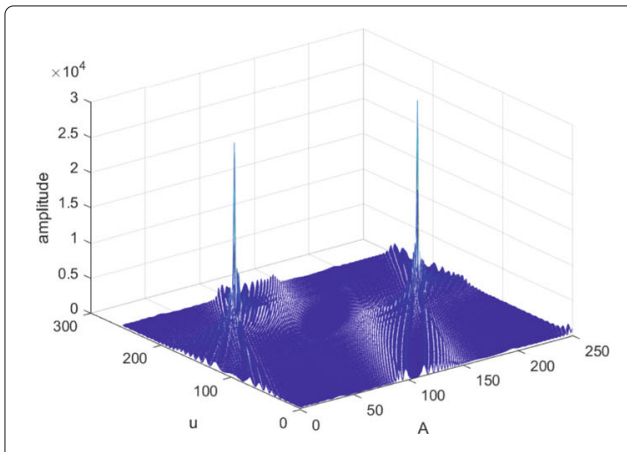

(a) IWVD

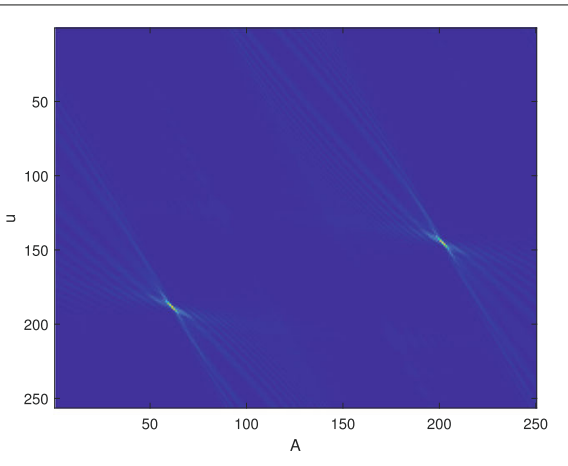

(b) IWVDL in $u-A$ plane

Fig. 7 IWVDL of two-component LFM signal 


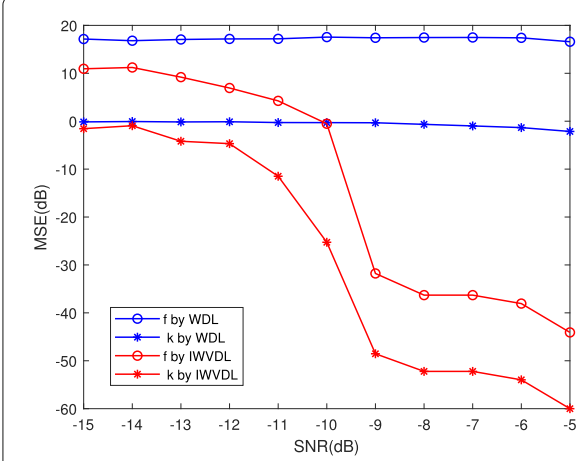

(a) mono-component LFM signal

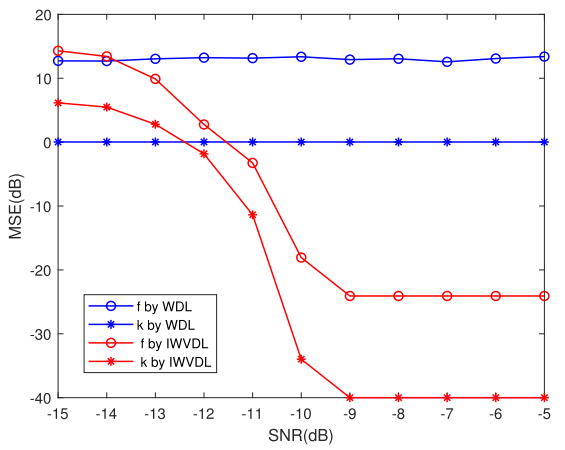

(b) multi-component LFM signal

Fig. 8 MSE of phase coefficient

where $L$ is the number of scatterer in a range bin, and $T_{a}$ is observation time. $C_{i}$ is backward reflection coefficient, and $a_{i 1}$ and $a_{i 2}$ denote effective rotation velocity and accelerated velocity of scatterer $i$ respectively.

Based on proposed IWVDL method, the parameters of each LFM signal in (45) can be estimated and cross term can be suppressed effectively. Figure 9 gives the ISAR imaging procedure based on proposed method. Associated with range-instantaneousDoppler imaging technique, the new ISAR imaging algorithm will be studied to obtain the high-resolution ISAR image in our future work.

\section{Conclusion}

Jointing advantage of WVD and LCT in time-frequency plane, this paper derives a WVDL from a new autocorrelation function in LCT domain and investigate other useful properties. For LFM signal, this WVDL can remove coupling between time and time delay, which helps estimate parameter of LFM signal. Based on decoupling characteristics, an integration form of WVDL is proposed to enhance energy and suppress cross term. Then, two phase parameters can be estimated simultaneously to avoid error propagation. Moreover, the simulations are carried out by mono-component and multi-component LFM signal, which shows good ability on parameter estimation and suppression of cross term. Finally, the superior performance is verified by comparison and discussion of the estimation error and computational cost. The application on ISAR imaging field will be are studied in the future work.

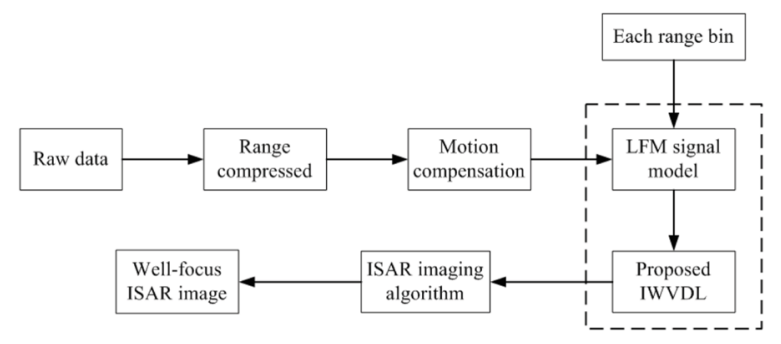

Fig. 9 Application to ISAR imaging 


\section{Abbreviations}

WVD: Wigner-Ville distribution; FT: Fourier transform; FRFT: Fractional Fourier transform; LCT: Linear canonical transform; UWDL: Unified WVD in the LCT domain; LCF: Linear canonical frequency; WVD: Wigner-Ville distribution associated with linear canonical transform; LFM: Linear frequency modulation

\section{Acknowledgements}

The author is very thankful to all colleagues and reviewers for their help and useful suggestions on this paper.

\section{Authors' contributions}

$\mathrm{H}-\mathrm{CX}$ contributed to propose newly and specially defined WVDL and derive its useful properties. Besides, H-CX contributed to simulation and manuscript by softwares. B-ZL contributed to the conception of new WVDL and review. Moreover, B-ZL contributed to the funding support. The authors read and approved the final manuscript.

\section{Authors' information}

Hong-Cai Xin was born in Handan, China, in 1991. She received the B.S. degree from Hebei Normal University, Shijiazhuang, China, in 2015. She is currently working towards the Ph.D. degree in Beijing Institute of Technology, Beijing, China. Her research interests include the theory and applications of fractional Fourier transform and linear canonical transform, inverse synthetic aperture radar (ISAR) and interferometric ISAR (InISAR), and time-frequency analysis. Bing-Zhao Li was born in 1975. He received the B.S. degree from Shandong Normal University in 1998, and the M.S. and Ph.D. degrees from Beijing Institute of Technology, in 2001 and 2007, respectively. He is currently a Professor with the School of Mathematics and Statistics, Beijing Institute of Technology, Beijing, China. He was a recipient of National Excellent Doctoral Dissertation Award Nomination and Henry Fok Education Foundation Young Teacher Award in 2010 and also supported by the New Century Excellent Talents in University in 2012. He is currently the Vice Dean of the School of Mathematics and Statistics. His current research interests include the theory and applications of fractional Fourier transform, linear canonical transform, and fractional calculus. He has published one book and more than 40 peer-reviewed journal articles.

\section{Funding}

This work was supported by the National Natural Science Foundation of China [No. 61671063] and China Scholarship Council [No. 201906030148].

Availability of data and materials

Please contact the authors for data requests.

\section{Declarations}

\section{Consent for publication}

This research does not contain any individual person's data in any form (including individual details, images, or videos).

\section{Competing interests}

The authors declare that they have no competing interests.

Received: 24 February 2021 Accepted: 22 June 2021

Published online: 03 August 2021

\section{References}

1. K. Gröchenig, Foundations of Time-Frequency Analysis. (Birkhäuser, Boston, Mass, USA, 2001)

2. D. Dragoman, Applications of the Wigner distribution function in signal processing. EURASIP J. Adv. Signal Process. 2005, 264967 (2005)

3. L. Debnath, B. V. Shankara, N. Rao, On new two dimensional Wigner-Ville nonlinear integral transforms and their basic properties. Integr. Transf. Spec. F. 21(3), 165-174 (2010)

4. C. Y. Mei, A. Z. Sha'ameri, B. Boashash, Efficient phase estimation for the classification of digitally phase modulated signals using the cross-WVD: a performance evaluation and comparison with the S-transform. EURASIP J. Adv. Signal Process. 2012, 65 (2012)

5. Y. S. Wu, X. K. Li, Elimination of cross-terms in the Wigner-Ville distribution of multi-component LFM signals. IET Signal Process. 11(6), 657-662 (2017)

6. H. N. Su, J. M. Pan, Q. L. Bao, Z. P. Chen, Parameter estimation of linear frequency modulated signals based on a Wigner-Ville distribution complex-valued convolutional neural network. J. Appl. Rem. Sens. 14(3), 036512 (2020)

7. R. Tao, B. Li, Y. Wang, G. K. Aggrey, On sampling of bandlimited signals associated with the linear canonical transform. IEEE Trans. Signal Process. 56(11), 5454-5464 (2008)

8. B. Z. Li, T. Z. Xu, Linear canonical transform and application. (Science Press, China, 2013)

9. Y. Sun, B. Z. Li, Sliding discrete linear canonical transform. IEEE Trans. Signal Process. 67(7), 4553-4563 (2018)

10. N. Goel, K. Singh, R. Saxena, A. K. Singh, Multiplicative filtering in the linear canonical transform domain. IET Signal Process. 10(2), 173-181 (2016)

11. X. Huang, L. R. Zhang, S. Y. Li, Y. H. Zhao, Radar high speed small target detection based on keystone transform and linear canonical transform. Digit. Signal Process. 82, 203-215 (2018)

12. J. Shi, X. Liu, X. J. Fang, X. J. Sha, W. Xiang, Q. Y. Zhang, Linear canonical matched filter: theory, design, and applications. IEEE Trans. Signal Process. 66(24), 6404-6417 (2018)

13. Y. N. Zhang, B. Z. Li, N. Goel, S. Gabarda, Quantitative SNR analysis of QFM signals in the LPFT domain with Gaussian windows. Sci. China Inform. Sci. 62(2), UNSP 15 (2019) 
14. S. C. Pei, J. J. Ding, Relations between fractional operations and time-frequency distributions, and their applications. IEEE Trans. Signal Process. 49(1), 1638-1655 (2001)

15. R. F. Bai, B. Z. Li, Q. Y. Cheng, Wigner-Ville distribution associated with the linear canonical transform. J. Appl. Math. 2012, $740161(2012)$

16. D. Y. Wei, Y. M. Li, Linear canonical Wigner distribution and its application. Optik. 125(1), 89-92 (2014)

17. Y. E. Song, X. Y. Zhang, C. H. Shang, H. X. Bu, X. Y. Wang, The Wigner-Ville distribution based on the linear canonical transform and its applications for QFM signal parameters estimation. J. Appl. Math. 2014, 516457 (2014)

18. Z. C. Zhang, M. K. Luo, New integral transforms for generalizing the Wigner distribution and ambiguity function. IEEE Signal Process. Lett. 22, 460-464 (2015)

19. Z. C. Zhang, Unified Wigner-Ville distribution and ambiguity function in the linear canonical transform domain. Signal Process. 114, 45-60 (2015)

20. Z. C. Zhang, New Wigner distribution and ambiguity function based on the generalized time shift in the linear canonical transform domain. Signal Process. 118, 51-61 (2016)

21. Z. C. Zhang, Novel Wigner distribution and ambiguity function associated with the linear canonical transform. Optik. 127(12), 4995-5012 (2016)

22. D. Urynbassarova, B. Z. Li, R. Tao, Convolution and correlation theorems for Wigner-Ville distribution associated with the offset linear canonical transform. Optik. 157, 455-466 (2018)

23. L. Y. Zheng, D. Shi, J. Zhang, CAF-FrFT: a center-affine-filter with fractional Fourier transform to reduce the cross-terms of Wigner distribution. Signal Process. 94, 330-338 (2014)

24. Z. Z. Guan, G. K. Xia, Q. Meng, Signal and linear systems. (Higher Education Press, Beijing, 2004)

25. B. Deng, R. Tao, Y. Wang, Comments on a convolution and product theorem for the linear canonical transform. IEEE Signal Process. Lett. 17(6), 615-616 (2010)

26. D. Y. Wei, Y. M. Li, Convolution and multichannel sampling for the offset linear canonical transform and their applications. IEEE Trans. Signal Process. 67(23), 6009-6024 (2019)

27. M. Huber, L. Czeslaw, D. Igor, Estimating the instantaneous frequency of linear and nonlinear frequency modulated radar signals-a comparative study. Sensors. 21 (8), 2840 (2021)

28. M. D. Xing, R. Wu, Y. C. Li, Z. Bao, New ISAR imaging algorithm based on modified Wigner-Ville distribution. IET Radar Sonar Navig. 3(1), 70-80 (2008)

29. Y. Wang, Y. Lin, ISAR imaging of non-uniformly rotating target via range-instantaneous-Doppler-derivatives algorithm. IEEE J. Sel Top. Appl. Earth Obs. Remote Sens. 7(1), 167-176 (2014)

30. J. B. Zheng, H. Liu, Q. H. Liu, Parameterized centroid frequency-chirp rate distribution for LFM signal analysis and mechanisms of constant delay introduction. IEEE Trans. Signal Process. 65(24), 6435-6447 (2017)

\section{Publisher's Note}

Springer Nature remains neutral with regard to jurisdictional claims in published maps and institutional affiliations.

\section{Submit your manuscript to a SpringerOpen ${ }^{\circ}$ journal and benefit from:}

- Convenient online submission

- Rigorous peer review

- Open access: articles freely available online

- High visibility within the field

- Retaining the copyright to your article

Submit your next manuscript at $\boldsymbol{\triangleright}$ springeropen.com 